\title{
Pituitary Infarct Masquerading as a Pituitary Abscess
}

\author{
Hyun Seok Choi, Bom-yi Kim, Yong-Kil Hong
}

Key words: neuroimaging, pituitary

A 64-year-old woman presented with headache for a week. Contrast-enhanced T1-weighted sellar magnetic resonance imaging showed an ovoid intrasellar nonenhancing lesion with peripheral enhancement (Figure 1). The lesion showed intermediate to high signal intensity on diffusion-weighted imaging and restricted diffusion on apparent diffusion coefficient map (Figure 2). Initial differential diagnosis included pituitary abscess and infected Rathke's cleft cyst. Initially, partial removal of the pituitary gland was performed via the transsphenoidal approach. The result of the frozen biopsy was pituitary infarct; thus, the operation was finished. The final pathological diagnosis was also pituitary infarct. Pituitary lesions with intermediate to high signal on diffusion-weighted imaging include pituitary abscess and pituitary infarct. ${ }^{1,2}$ A pituitary infarct should be considered as a differential diagnosis in the case of pituitary apoplexy.

\section{Disclosures}

None.

\section{REFERENCES}

1. Takayasu T, Yamasaki F, Tominaga A, Hidaka T, Arita K, Kurisu K. A pituitary abscess showing high signal intensity on diffusionweighted imaging. Neurosurg Rev. 2006;29:246-8.

2. Rogg JM, Tung GA, Anderson G, Cortez S. Pituitary apoplexy: early detection with diffusion-weighted MR imaging. AJNR Am J Neuroradiol. 2002;23:1240-5.
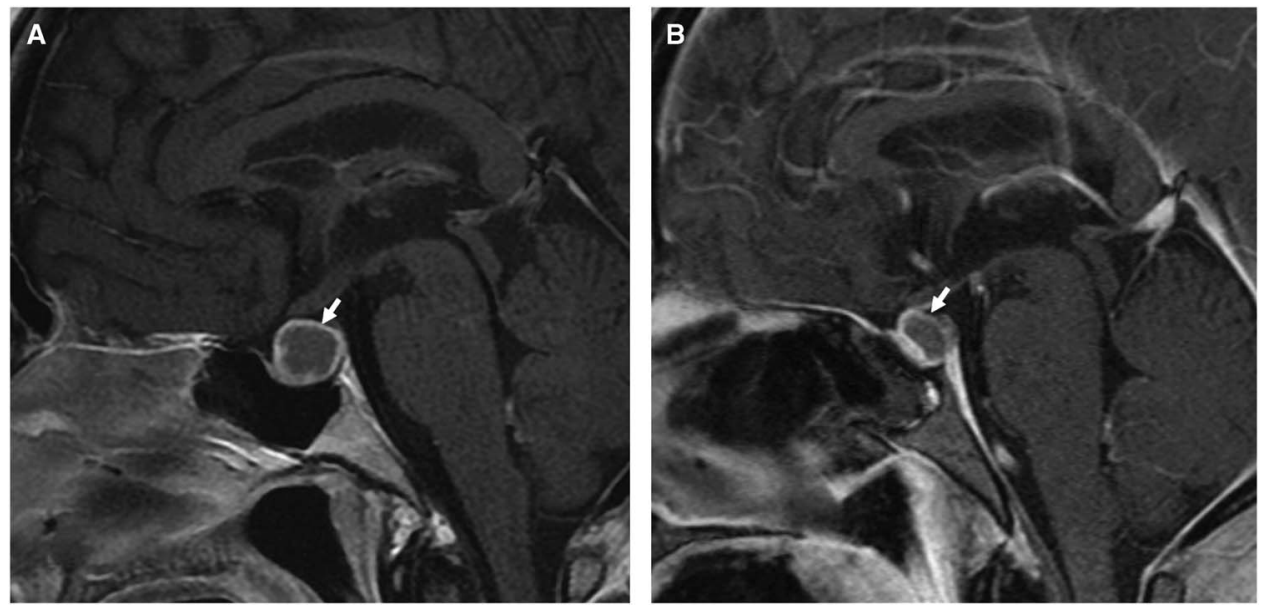

Figure 1: (A) Preoperative sagittal contrast-enhanced T1-weighted image shows enlarged pituitary gland with a thin peripheral rim enhancement (arrow). (B) Postoperative contrast-enhanced T1-weighted image shows a decrease in size of the pituitary lesion (arrow).

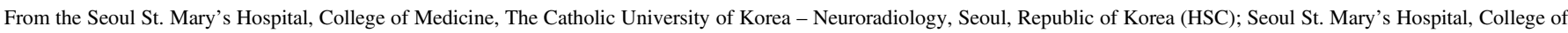
Medicine, The Catholic University of Korea - Neuroradiology, Seoul, Republic of Korea (BK); Seoul St. Mary's Hospital, College of Medicine, The Catholic University of Korea -

Neurosurgery, Seoul, Republic of Korea (YKH)

Received October 17, 2014. FinAl Revisions Submitted June 22, 2015.

Correspondence to: Hyun Seok Choi, Seoul St. Mary's Hospital, College of Medicine, The Catholic University of Korea - Neuroradiology, Seoul, Republic of Korea.

Email: hschoi@catholic.ac.kr. 

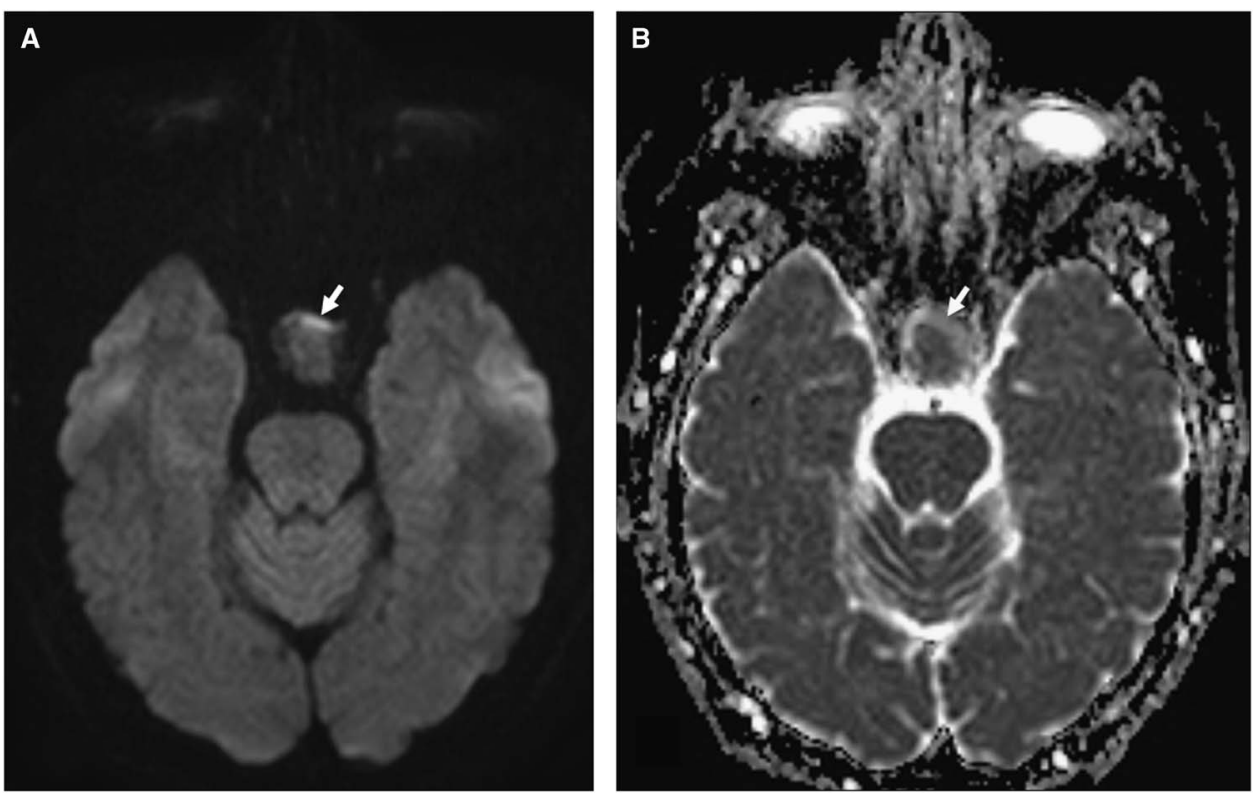

Figure 2: Diffusion weighted imaging (A) and apparent diffusion coefficient $(A D C)$ map $(B)$. The lesion showed intermediate to high signal intensity on diffusion-weighted imaging and restricted diffusion on ADC map (arrows). This phenomenon is known as pseudonormalization, in which high signal intensity on diffusion-weighted imaging begins to vanish in 1 week after acute infarct. The imaging time point of this patient was 2 weeks after symptom onset. 